University of South Florida

DIGITAL COMMONS

Digital Commons @ University of

@ UNIVERSITY OF SOUTH FLORIDA

South Florida

Rehabilitation and Mental Health Counseling

Faculty Publications

Rehabilitation and Mental Health Counseling

2007

\title{
High-Speed Networking and Embedded Gerontechnologies
}

\author{
William D. Kearns \\ University of South Florida, kearns@usf.edu \\ James L. Fozard \\ University of South Florida
}

Follow this and additional works at: https://digitalcommons.usf.edu/mhs_facpub

\section{Scholar Commons Citation}

Kearns, William D. and Fozard, James L., "High-Speed Networking and Embedded Gerontechnologies" (2007). Rehabilitation and Mental Health Counseling Faculty Publications. 59.

https://digitalcommons.usf.edu/mhs_facpub/59

This Article is brought to you for free and open access by the Rehabilitation and Mental Health Counseling at Digital Commons @ University of South Florida. It has been accepted for inclusion in Rehabilitation and Mental Health Counseling Faculty Publications by an authorized administrator of Digital Commons @ University of South Florida. For more information, please contact digitalcommons@usf.edu. 


\title{
High-speed networking and embedded gerontechnologies
}

\author{
William D. Kearns PhD \\ Internet2 University Executive Liaison, Department of Aging and \\ Mental Health, Louis De La Parte, Florida Mental Health Institute, \\ University of South Florida, Tampa, Florida 33612-3807, USA \\ E: kearns@fmhi.usf.edu
}

\author{
James L. Fozard PhD \\ School of Aging Studies, University of South Florida \\ Tampa, FL 33612, USA
}

W.D. Kearns, J.L. Fozard, High-speed networking and embedded gerontechnologies. Gerontechnology 2007; 6(3):135-146. Information and communications technologies (ICT) have undergone extraordinary advances in the past 5 years. Two aspects of these developments are described along with their implications for gerontechnology. First, international high speed Internet networks serving the academic community are reviewed, acknowledging that today's academic networks are poised to inform the public's computer networks of tomorrow. Second, computational systems that increasingly penetrate every facet of the built environment, for instance, clothing, furnishings, personal items, transportation and home environmental control and security are described. Over time such embedded systems are integrated into large wired and wireless networks of devices which may extend over international boundaries. High bandwidth networked applications in development include multipoint videoconferencing using multicast IPv6 protocols, telepresence, virtual reality simulations and remote sensing for gathering data in built environments. Examples of how these developments create new applications for all four of the goals of gerontechnology - prevention, compensation, care and enhancement of quality of life - are provided. Enhanced technological services for the elderly will develop, including nutritional monitoring, safety and security, mental health and healthcare, environmental control, and communications. International networks promise more uniform care standards for the elderly, increase the opportunity for collaboration among researchers and educators tackling the difficult problems associated with aging, including dementia, heart disease, diabetes and obesity. Networks have the promise of enhancing outcomes by maximizing economies of scale by collecting research observations from multiple international venues. New educational approaches addressing the challenges of aging include virtual reality applications which simulate the impact of aging for young individuals. Monitoring individuals in their homes using ICT and computer networks can impose significant ethical responsibilities upon governing agencies.

\section{Keywords: Internet evolution, embedded ICT, gerontechnology services}

In this paper we review the rapid escalation in speed and bandwidth of the internet, two developments that have resulted in very high speed worldwide computer networks. We also review the parallel, rapid evolution of computational devices which allow information transfer amongst people and embedded systems in the built environment, for instance, clothing, personal items, and appliances. Separately and in combination, the implications of these developments for a broad range of gerontechnology applications and development are discussed.

\section{EvOLUTION OF THE INTERNET}

The inception of the internet may be traced to October 29 ${ }^{\text {th }}, 1969$ when Dr. Leonard 


\section{High-speed networking}

Kleinrock, then an Associate Professor of Engineering at the University of California at Los Angeles, created the first networked computer connection between a computer in his laboratory and a computer at the Stanford Research Institute (SRI) ${ }^{1}$. The experiment, consisting of two computers was technically a failure, resulting in the SRI machine crashing before the login step was completed. Undaunted, these scientists redoubled their efforts and eventually succeeded in creating the first local and then wide area networks (LANs and WANs). Continued funding by the US Department of Defense's Defense Advanced Projects Administration (DARPA) created ARPANET, which was heavily used during the Cold War period for atomic energy research among DOD sponsored research centers at universities, giving rise to NSFNET (the National Science Foundation Network), a peacetime application of computer networking to scientific research and applications.

ICT (information and communications technology) is an umbrella term that includes any communication device or application encompassing radio, television, cellular phones, computer and network hardware and software, satellite systems and so on, as well as the various services and applications associated with them, such as videoconferencing and distance learning. This civilian use of ICT is widely accepted as the point in time where the internet was created, because scientific research was already being facilitated by international computer networks spanning the globe; indeed, e-mail began to be regularly transmitted among scientists in 1972. The general public was denied access to this costly network and appropriate use policies were strict. The scientific applications running on these networks were often complex, custom written programs, which took significant time and effort to learn and use. Software development was more of a cottage industry with programmers laboriously writing code for specific applications and modifying software by hand to meet the specific needs of their research.

In 1991, the privatization of the internet allowed citizens direct access to the network and they began to communicate with others across great distances. However, in 1993 a world-shaking event happened with the release of the first graphical web browser, 'Mosaic' from the National Center for Supercomputer Applications ${ }^{2}$ and CERN (Conseil Européen pour la Recherche Nucléaire: the European Organization for Nuclear Research). Mosaic integrated difficult to use technologies, such as 'Telnet (an openly accessible public packet data service that allows a computer operator at a terminal or PC to log onto a remote computer and run a program)', and 'FTP' (File Transfer Protocol), and an increasing array of other tools, in a simple interface that enabled even the most unsophisticated user to surf the internet with comparative ease. The coincident privatization of the internet meant anyone could create a World Wide Web site capable of providing services to visitors, leading to a proliferation of sites now numbering in the millions. Local connection speeds, however, were slow, averaging only a few hundred bits per second (baud). Coincident with website proliferation was the proliferation of headaches in the forms of viruses, worms, Trojan horses, phishing scams, identity fraud, and other problems characteristic of illicit communication activities in general.

Mosaic's creators retired from academe and started Netscape, Inc. Home network speeds increased from 300 baud to 54.000 baud over telephone modems, and 750.000 baud access over digital subscriber lines (DSL), and 10.000.000 baud over cable modems to the home. These technologies are currently being supplanted by $30 \mathrm{Mbit} / \mathrm{sec}$ fiber optic network service to the home. With each major increment in speed, new classes of applica- 
tions have become available. High definition digital video is the 'gold standard' $d u$ jour but will likely be short-lived as newer technologies are brought to market.

\section{HIGH SPEED COMPUTER NETWORK DEVELOPMENT}

Universities were crucial to the creation of the internet. Stanford's pioneering work enabled the creation of DARPANET, NSFNET, and the internet. Universities have continued to be major driving forces in internet development since the internet's privatization. In 1996, 34 American universities joined together to create the University Corporation for Advanced Internet Development (UCAID) $)^{3}$. The goal of UCAID was to tap universities' creative power to reinvent the internet by imagining and building whole new classes of applications previously undreamt of. Inherent in UCAID's philosophy is that university laboratories should drive new network applications, which should move to the marketplace and steer the developing internet economy. This philosophy is codified in UCAID's Internet2 project, a consortium of over 200 of the most prestigious universities in the United States united in a common goal of advancing high speed internet development through university / corporate partnerships.

The strategic importance of high speed network development has been seized upon by other nations. Currently, the Internet2 project shares memoranda of understanding with over 26 other high speed networks whose sponsors have comparable objectives to Internet $2^{4}$. Within Western Europe, SurfNet brings high speed service to the Netherlands, Renater to France, Geant and Dante to Germany, NorduNet to Scandinavia and in the Americas, Canarie to Canada, and CUDI to Mexico, to name just a few of such networks. Each network offers connectivity to hundreds of universities in its region of coverage (Table 1).

The conditions of use for these networks vary and have evolved over time. For example, Internet2's 'Abilene' ${ }^{5}$ network forbade corporate use except for noncommercial research purposes with a university affiliate. Abilene's replacement, NewNet ${ }^{\prime \prime}$, will replace Abilene in September 2007, and will allow participants to use it commercially; meaning universities will gain a financial incentive to create applications and may invite corporate participation. This policy change is anticipated to stimulate Internet2 development and encourage other networks to adopt similar policies favorable towards free enterprise.

Within Internet2, the focus has consistently been on developing high bandwidth applications which stress networks to the breaking point in order to improve their resistance to failure and to achieve their full potential. Considerable attention has been paid to the development of the 'killer application', which to date has been digital video. Video is particularly demanding of network resources because of its ability to utilize almost limitless bandwidth and its requirements that packets (i) arrive in the order in which they were transmitted, and (ii) have very low latency in their arrival times and low jitter (the sending and receiving machines remain in synchronization). In contrast, applications, such as FTP and web pages, are comparatively undemanding. Internet2 applications currently under development include multipoint videoconferencing using IPv6, Telepresence, virtual reality, and remote sensing over great distances. Telepresence is a communications medium that enables a person to feel as if he or she is actually present in a different place. Telepresence utilizes technologies to enable people to experience and take action in real places that are physically distant, hazardous, or inaccessible. Virtual environments are computer-generated worlds that users may enter and take action in. These environments can facilitate the experience of being elderly for young persons. 


\section{High-speed networking}

Table 1. High speed international networks peered with Internet2

\begin{tabular}{|c|c|c|c|}
\hline Organization & Country & Organization & Country \\
\hline \multicolumn{2}{|c|}{ Americas } & \multicolumn{2}{|c|}{ Europe and the Middle East } \\
\hline CANARIE & Canada & ARNES & Slovenia \\
\hline CLARA & Latin America & BELNET & Belgium \\
\hline & \& Caribbean & CARNET & Croatia \\
\hline CEDIA & Ecuador & CESnet & Czech Republic \\
\hline CNTI & Venezuela & DANTE & Europe \\
\hline CR2Net & Costa Rica & DFN-Verein & Germany \\
\hline CUDI & Mexico & FCCN & Portugal \\
\hline REUNA & Chile & GARR & Italy \\
\hline RETINA & Argentina & GIP-RENATER & France \\
\hline RNP [FAPESP] & Brazil & GRNET & Greece \\
\hline SENACYT & Panama & HEAnet & Ireland \\
\hline \multicolumn{2}{|c|}{ Africa, Asia and the Pacific } & HUNGARNET & Hungary \\
\hline AAIREP & Australia & Israel-IUCC & Israel \\
\hline APAN & Asia-Pacific & MCIT (EUN/ENSTINET) & Egypt \\
\hline ANF & Korea & NORDUnet & Nordic countries \\
\hline $\begin{array}{l}\text { CERNET, CSTNET, } \\
\text { NSFCNET }\end{array}$ & China & $\begin{array}{l}\text { PSNC/PIONIER } \\
\text { (was POL-34) }\end{array}$ & Poland \\
\hline CDAC, ERNET & India & Qatar Foundation & Qatar \\
\hline JAIRC & Japan & RedIRIS & Spain \\
\hline JUCC & Hong Kong & RESTENA & Luxemburg \\
\hline MYREN/MDeC & Malaysia & RIPN & Russia \\
\hline NECTEC/UNINET & Thailand & SANET & Slovakia \\
\hline REANNZ & New Zealand & Stichting SURF & Netherlands \\
\hline SingAREN & Singapore & SWITCH & Switzerland \\
\hline TANet2 & Taiwan & TERENA & Europe \\
\hline TENET & South Africa & UKERNA, JISC & United Kingdom \\
\hline
\end{tabular}

\section{People ANd computer transfers}

The development of high-speed networks has paralleled an equally striking maturation of embedded computer systems appearing in handheld devices and household appliances. Formerly 'dumb' machines, such as refrigerators, are being redesigned with computers to make them more energy efficient, and in the future will enable them to reveal their contents to their owners as they search their favorite grocery store for foodstuffs ${ }^{7}$. With the drop in computing costs and the doubling of computing power every 18 months in accordance with Moore's Law, intelligent machines are becoming the norm rather than the exception. As a case in point, one of the authors' home environmental control systems was replaced with a computerized version of the simple but trustworthy bimetallic strip used to switch the environmental control system on and off. The replacement immediately netted a $10 \%$ savings in electricity costs per month and allowed for complex scheduling of room temperatures contingent on the presence of persons in the home, which resulted in yet further savings. More recent versions of the environmental control systems can be telephoned or accessed via the internet and reprogrammed remotely by the owner ${ }^{8}$.

Data exchange among appliances provides numerous avenues for improved service integration. Appliances can now use wireless protocols, such as Bluetooth, ZigBee, and 802.11 Ethernet, to exchange information. One might envision an alarm clock that communicates wirelessly to the user's coffee maker and cell phone. The coffee maker will only make coffee when the 
user turns off the alarm, but will not make coffee when the user presses the 'snooze' button indicating his desire to continue sleeping. Likewise his cell phone's ringer would also not turn back on until the user permanently switched off the alarm, signaling that he was starting his day and willing to accept incoming calls.

\section{New Gerontechnologies}

High-speed networks create opportunities for widespread monitoring and service delivery for individuals, often at extraordinary distances. They may expand applications in all five domains of gerontechnology Health \& Self-esteem, Housing \& Daily Activities, Communication \& Governance, Mobility \& Transport, and Work \& Leisure. The goals of gerontechnology interventions - the prevention or delay of age-related losses of function, compensation for functions that decline with age, care for elderly persons with disabilities, and enhancement of the quality of life - will all have expanded possibilities as a result of the developments described above ${ }^{9}$. The examples to be discussed show how high speed networking will provide the basis for expanded services that contribute to independence and well-being of aging and aged people. High speed communication between a person and several smart technologies, which themselves may be networked, is the main feature of networking that underlies the many examples to be cited.

\section{Monitoring and interventions}

For goals related to compensation and care in the Health and Self-esteem domain, high-speed networks enhance telemedicine applications, such as videoconferencing and telehealth (remote monitoring of physiological conditions), by reducing packet latency and jitter and improving overall quality of service for these applications. Low packet latency and jitter are essential to the proper diagnosis and treatment at a distance of movement disorders, such as Parkinson's disease. The activities of persons outside and inside their homes can be monitored using global positioningbased location and context aware applications and wayfinding tools such as the Activity Compass which was developed for persons with dementia by Patterson, Etzioni, and Kautz ${ }^{10}$ and which utilizes wireless networks.

Radio Frequency Identification (RFID) technologies offer some of the greatest opportunities for integration into large scale networks in the near future. A variant under investigation is ultra-wideband RFID, which uses powered transponder devices (referred to as tags) to communicate with base stations over distances of as much as 183 meters and may use wireless internet protocols (IP) as a communications channel. Tag position can be determined to the decimeter range anywhere in the field of view of the receiving antennae, making the tags extremely useful for providing location-aware services contingent on a person's position in the home ${ }^{11}$. Because the tags are tiny computers, they may acquire data directly from the wearer, including physiological measures, acceleration information, etc. Their theoretical bandwidth for transferring data exceeds $1 \mathrm{Gi}$ gabit/sec at close range ${ }^{12}$. The battery life on such tags may exceed two years under normal use, permitting extended service provisioning and monitoring. The application of RFID in home settings is particularly useful in situations requiring differentiating people within a common locale. For example, appliances can be customized to the desires of multiple users in the home and can learn the user's identity by the proximity of their unique tag. Interactive movements of persons with dementia and their caregivers may be studied to better understand wandering behaviors ${ }^{13}$. Similarly, the interactions of new nursing home admissions with a service robot may be monitored to assess how well they are adapting to the novel environment. This strategy has been utilized by Japanese researchers ${ }^{14}$ employing 'Paro', a 


\section{High - spe ed networking}

robotic harp seal whose behavior adapts to the nursing home resident (Figure 1). As it learns, Paro provides researchers with data about the person's adaptation to the nursing home environment via sensors embedded in its skin and its wireless network connection. At least one study of nursing home residents has shown reduced stress hormone levels in elders when Paro learns from its social interactions with them, but when Paro engaged in stereotyped behaviors unaffected by elder interaction levels of stress hormones remained elevated ${ }^{14}$. High-speed network links for robots may increase their behavioral versatility; machine to machine communication permits distributed intelligence allowing individual robots to cache the minimum information necessary to perform their function and yet benefit from system wide changes in care policies.

140 As indicated above, networking allows for remote monitoring of an individual's activities in a home setting that goes far beyond the level provided by services such as LifeLine ${ }^{\circledR}$ or LifeAlert ${ }^{\circledR}{ }^{15}$. The movements and location of a person can be tracked, and the use of food, water, heat, electricity, etc., may be determined through networks with other smart technologies. In home environments, monitoring the quality of indoor air is particularly important for elderly persons with pulmonary or cardiopulmonary disease. Deviations from normal behavior patterns within the home environment may indicate problems with gait, breathing, etc. The sensors required for such applications can be embedded in articles of clothing or wearable appliances ${ }^{16}$ thereby increasing the potential for monitoring outside of home settings - to anywhere a receiving antenna is available.

\section{Improved interventions}

High-speed networks allow faster interactions between a person and remote machines in games, virtual simulations, machine-guided physical rehabilitation

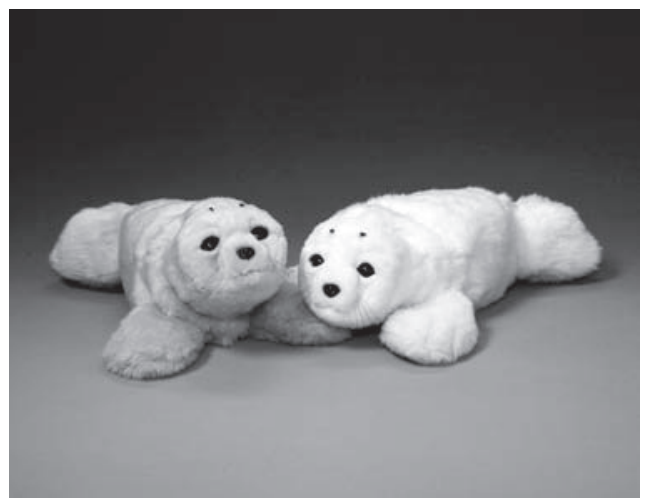

Figure 1. Paro the Robotic Harp Seal with Adaptive Intelligence ${ }^{14}$

activities, detection of dangerous changes in gait, identification of dangerous changes in driving behavior, etc., all applications that are particularly relevant to gerontechnology goals of prevention and enhancement. In this respect high-speed networks are analogous to the body's nervous system and provide sensory and effector data as well as serving an integrative function with ICT. Smart gerontechnologies should perform their functions more efficiently than their 'non-smart' alternatives. If we conceptualize a given gerontechnology as a human interface with a network, we can consider how differing intelligence levels in the interface and the larger network can affect interactions with the elder user. For example, eyeglasses are a technology that improves an elder's ability to gain information from and to interact with their environment. However, eyeglasses tend to be 'dumb' since additional information from an outside source may not (at least not at present) improve their performance. In this instance, the eyeglass interface has no intelligence. In contrast, consider an electronic hearing aid with sophisticated acoustic profiling software built in and having Bluetooth connectivity to networked resources ${ }^{17}$. As a computing machine, the hearing aid is a remarkable device. Its local computing power, however, may be augmented efficiently by interfacing it to a wide area network capable of delivering software upgrades 
to the operating system of the hearing aid via Bluetooth. The designer must decide the amount of intelligence to reside in the interface (hearing aid), and this choice must be informed by at least three parameters. First, the hearing aid's level of desired autonomy must be determined by the designer. If the device must operate in isolation from a wide area network for extended intervals, it should have enough intelligence to be self-sufficient. Second, if the hearing aid must have its operating system patched or upgraded regularly to enhance its performance for the benefit of the elder whose hearing may be deteriorating quickly, then access to a fast network connection would be very beneficial to effect these changes, however, a lower premium may be placed on self-sufficiency. A third factor to be determined by the gerontechnology designer is the degree to which a device must actively process data and reflexively respond to changing local circumstances, ambient noise in the case of hearing aids. As a second example, a device which detects and corrects for abnormalities in gait may need to calculate geometry very quickly in order to prevent a fall and possible hip fracture. Fast network response speeds could be offset by unpredictable network congestion; however, this might be less a factor on very high-speed computer networks. A hip fracture caused by data processing delays at a remote computer would be a very bad outcome. In this instance, local processing speed would be prized so dynamic changes in the elder's position could be rapidly compensated.

\section{Consumer activities}

In addition to its current uses in inventory control, RFID technology is becoming more integrated in customer oriented retailing operations. This is a development relevant to gerontechnology's goal of enhancement. Because RFID devices are small computers, the information they provide can duplicate the information carried on bar codes, thereby simplifying the purchase of products through self- or clerk-run checkout operations. A person's purchasing history can be used by the retailer to advertise related products or promote the sale of less expensive alternatives to those in the customer's shopping cart. The customer may also use networks to learn about a rival store's prices for the same items ${ }^{18}$. RFID tags may be linked in novel ways to electronic display devices to expand the presentation modalities for product information to compensate for age associated declines in perceptual and motor function, not a trivial matter. Roger Coleman, an industrial designer, vividly illustrated the problems encountered by older persons shopping in British Safeway Stores by giving members of the Company's Board of Directors-mostly elderly themselves--a shopping list of items to purchase in a typical Safeway Store ${ }^{19}$. The directors were greatly surprised at how difficult it was to read labels and reach items on shelves, and they instructed store managers to take steps to make it easier for older persons to shop at Safeway Stores ${ }^{19}$.

Internet on-line comparison shopping and product evaluation is currently the norm. High-speed networking moves these activities from the desktop PC to the handheld cellular telephone in the center of the marketplace. A customer can scan a product and see immediately on his telephone's display what it costs in a competitor's store down the street ${ }^{20}$. Consumer networks of phone shoppers can share product data among themselves thereby optimizing their buying power, important to persons on a fixed income. Networking senior customers will more readily obtain competitive prices on products used by all age groups, but especially those targeted towards them. Aside from using networks to find the best prices, senior customers can adopt a practice already in vogue with teenagers; a girl trying out new outfits can use her cell phone camera to model them for her friends who can advise her on the best choice ${ }^{20}$. 


\section{Coaching, guidance and motivation}

The way that machines influence human behavior has evolved with changes over time in the natural and built environment (secular changes). Man's struggle to master the machine has been the topic of humorous films such as 'Modern Times', featuring Charlie Chaplin's struggle with an unruly assembly line, and the French film, 'Mr. Hulot's Holiday', showing the star battling a cantankerous automobile. A new use of computers to influence human behavior has been formally described in a recent book, 'Persuasive Technology'21. Machines can provide coaching, guidance, and reinforcement for specific behaviors that may fulfill gerontechnology goals. Precisely timed structured guidance and wayfinding instructions can be provided to tourists and others via high speed networks. One application of smart networked technology to manage wandering behavior of persons with dementia has been proposed ${ }^{22}$. Location aware and communication technology on the wanderer would be linked to provide synthetic voice instructions to the person; the messages transmitted would be contingent on the location information.

Motivation for establishing and maintaining healthy lifestyles throughout life is a challenge for people of all ages but is especially challenging for seniors. Cardiovascular and strength training machines provide opportunities for reinforcing behavior based on user performance ${ }^{23}$. The desired behavior in cardiovascular training is the maintenance of a target heart rate for a given period of time. Feedback consisting of heart rate, distance traveled, walking speed, energy expended, and even the display flashing 'Great workout!' at the workout's conclusion are all designed to reinforce exercise behavior. Networked exercise technology opens up additional opportunities for reinforcement including facilitating competition among peers performing similar exercises; individuals may be motivated to exercise harder in order to change their ranking among athletes. Similar approaches may benefit weight training where networked exercise technology helps identify target performance for everyday users. 'Smart' clothing, containing embedded sensors and systems that provide information about energy expended or a similar measure of muscle group performance, might provide a better training guide than the number of times a given weight is $\operatorname{lifted}^{16}$. Such a development might be of special interest to women, who may not be so interested in increasing visible muscle mass in the way many men are but are instead interested in maintaining muscle tone.

Networks can promote artistic and social activities among seniors, everything from virtual quilting bees to virtual jam sessions-musical and similar non-musical activities. Networking increases the opportunities for both serial and parallel additions to a work of art by network members perhaps even more so than was possible in the technology-facilitated group painting activity described by Bouma and Harrington ${ }^{24}$.

\section{ETHICS AND SECURITY}

The issue of security and confidentiality of information streams generated by networked gerontechnologies in the home is significant, yet in some sense the argument against networking gerontechnologies has been rendered moot by the excessive amount of personal information already available to the casual observer of the Internet. A person skilled in data mining can reconstruct much of our daily behavior patterns using our credit card purchase histories, credit histories, online government documents, digital video recordings of our business transactions, and the myriad internet sites we voluntarily fill with our personal information. To a large extent adding networked gerontechnology to a home gives only a trivial advantage to individuals who are intent on using such information for criminal activities. Increas- 
ingly stringent standards are evolving for the protection of information transferred by computer networks; the data are automatically encrypted and sent to remote hosts via secure communications channels ${ }^{25}$. Middleware protocols, such as Shibboleth, designed to protect critical data and medical records are being perfected at American universities and must comply with strict federal health privacy standards according to the HIPAA (Health Insurance Portability and Accountability Act) guidelines. The tiny loss of privacy risked by transmitting gerontechnology data streams is more than offset by the huge potential they provide for gathering new insights into the aging process and helping designers create better gerontechnologies and gerontechnology services.

Of greater concern is the potential for the spreading of viruses, worms, and other forms of what Hypponen ${ }^{26}$ calls 'malware' to mobile phones and other devices used to connect with the internet. Cellular phones are increasingly used to download programs and information from the internet and they are vulnerable to the same problems experienced by users of personal computers. Because they are wirelessly networked, the opportunistic virus caught by one phone can be more easily spread to other phones or other computing devices.

\section{ACCESSIBILITY AND USABILITY}

High-bandwidth networks such as Internet2's Abilene backbone are currently restricted to academic institutions and their partners only. US citizens cannot access these resources except through a relationship with a subscribing university or their partner institution; however, other nations' networks vary in their conditions of use and must be reviewed on an individual basis. The highest bandwidth networks by definition will always be in prototype stages but their product spin-offs will appear in the public domain as commercial offerings such as Verizon Inc's FIOS 30 Mbit connectivity to the home. With regard to usability, alternative protocols to Internet Protocol version 6 (IPv6) (the current highspeed standard) are always under development in university laboratories; however, there is a large investment in IPv6 as the successor to IPv4, the standard on which most networked applications are currently based. The specifications of the network protocol have a direct bearing upon the types of user applications that result. Usability becomes of paramount importance at the user application interface where the difficult human factors design work takes place, ensuring that the gerontechnology meets the needs of the elders it was designed to serve. An area of network research with direct implications for gerontechnology applications development relates to the implementation of multicast technologies ${ }^{27}$. High bandwidth multicast is not regularly or uniformly implemented on the commercial internet and remains largely experimental; however, the potential changes it may usher in when introduced to the public internet are enormous. Multicast permits a single network device to stream data to thousands of other devices simultaneously. A single video camera connected to the high-speed network becomes an international TV station carrying video worldwide to potentially thousands of viewers while not significantly increasing network traffic. In contrast, current video is sent using Unicast, which requires that the transmitter establish separate video streams to each receiver of the video, multiplying the amount of bandwidth consumed by the number of viewers. In a hypothetical gerontechnology application, the data from all of the interacting embedded systems monitoring an elder in a single household could be sent to hundreds of researchers worldwide simultaneously for analysis. This cannot be done currently without replicating the data stream for each researcher from each transmitting device, at a high cost in both bandwidth and hardware resources. 


\section{R\&D APPLICATIONS}

Compared to cross-sectional studies of aging that infer age related changes from differences between age cohorts, serial observations of aging in the same individual are generally considered to provide superior scientific information about aging processes $^{28}$. We believe that the entire approach to longitudinal research on aging may be significantly altered by the highspeed networking technology under discussion. Physiological and behavioral information can be obtained easily in natural settings, thereby increasing the efficiency, comprehensiveness, and convenience of longitudinal research that currently requires volunteers to make periodic visits to specialized facilities. Moreover, the interactions between a person and his or her environment can be described at a level heretofore impossible, permitting operational definitions for concepts such as the balance of environmental press against personal competence that are embedded in many transactional theories of aging ${ }^{29-}$ 33. The expanded possibilities for gathering data about aging in natural settings in multiple cultures will require a considerable amount of work in formulating testable hypotheses, an exciting prospect for gerontologists.

The invention of the telescope enabled early explorers to peer into the heavens and record nature's heavenly bodies in exquisite detail. The clarity provided by the instrument allowed the precise plotting of astronomical paths and a better understanding of our universe. Today, massively networked astronomical arrays permit us to track thousands of bodies simultaneously, searching for new knowledge about the universe. Likewise, we have the opportunity to turn the telescope upon ourselves and learn by tracking not heavenly bodies, but human bodies as they age. Networked gerontechnologies scattered throughout the European Union, the Americas, Asia, and Africa can simultaneously provide services to seniors and record valuable in- formation which will assist their designers to construct better gerontechnologies capable of providing an ever widening and precisely defined array of services. The data collected will allow us to better understand the needs of aging populations, and the differences that exist among cultures. This last element is very significant insomuch as today's seniors are more mobile than ever before and may migrate across international borders when they retire. A better understanding of their behavior and gerontechnology service use in their culture of origin may assist the process of assimilation into their adopted culture.

\section{Conclusion}

This essay has described the evolution of the internet from its beginnings through the current developments in high-speed internet networks that are now in existence in most of the world. It also described parallel developments in the way computing capacity can be distributed in the built environment, thereby allowing for greater communication amongst people, smart appliances and products, and among the appliances and products themselves. With this background, gerontechnological applications were described that illustrate the myriad ways in which information exchanges between a person and their environment are possible as a result of high-speed networking. Networked technology provides people with the ability to easily obtain critical information about themselves as well as information about their environment and products they want and use. At the same time, coaching, medical and rehabilitative services that support healthy lifestyles and independent functioning of elderly persons can be provided in a natural home setting to a degree unimagined up to now. The developments described provide the potential for longitudinal observational studies of aging of people in natural settings, the results of which can form the basis for interventional research and cultural comparisons of aging. 


\section{Acknowledgements}

The writers wish to thank Ms Ardis Hanson, Dr. Victor Molinari and the anonymous reviewers of a previous version of this article for their constructive criticisms and suggestions. Some of the material contained in this article was presented at the International Conference on Gerontic Technology and Service Management Conference, Nan Kai, Taiwan - March 26, 2007 by William D. Kearns and James L. Fozard under the title of 'Persuasive Technologies which Change Attitudes and Behaviors Can Support the Ambitions and Activities of Older Persons'.

\section{References}

1. Leiner B, Cerf V, Clark D, Kahn R, Kleinrock L, Lynch D, Postel J, Roberts $\mathrm{L}$, Wolff $\mathrm{S}$. The past and future history of the internet: The science of future technology. Communications of the ACM 1997;40(2):102-108

2. National Center for Supercomputer Applications. The future frontier: Computing on NCSA Mosaic's $10^{\text {th }}$ anniversary; 2003; http://www.ncsa.uiuc. edu/Conferences/MosaicEvent/; retrieved January 26, 2007

3. Internet2. Ann Arbor: The Association; 2007; Internet2: International relations:

An overview;

http://international.internet2.edu/ resources/presentations/2006/20060808internet2intlpartners.pdf; retrieved: January 26, 2007

4. Internet2. Ann Arbor: The Association; 2007; Internet2 international partnerships; http://international.internet2.edu/ index.cfm; retrieved January 26, 2007

5. Internet2. Ann Arbor: The Association; 2007; Abilene: Advanced networking for leading-edge research and education; http://abilene.internet2.edu/; retrieved: January 26, 2007

6. Internet2. Ann Arbor: The Association; 2007; Internet2's new network backbone: Technical overview; http://www. internet2.edu/files/ Internet2-New-Network-Tech-v0.9.pdf; retrieved January 26, 2007

7. Helal S, Mann W, El-Zabadani H, King J, Kaddoura Y. The Gator Tech Smart House: a programmable pervasive space. Computer 2005;38(3):50-60

8. Carner GA, Hesse BJ, Schultz $\mathrm{KV}$. Apparatus and method for wireless control. United States patent US 6622925 B2. September 23, 2003

9. Bronswijk JEMH van, Bouma $H$, Fozard J. Technology for quality of life: An enriched taxonomy. Gerontechnology
2003;2(2):169-172

10. Patterson D, Etzioni $\mathrm{O}$, Kautz $\mathrm{H}$. The activity compass. In: Proceedings of UbiCog '02: First International Workshop on Ubiquitous Computing for Cognitive Aids, Gothenburg, Sweden. September 29, 2002; http://luci.ics.uci.edu/ websiteContent/weAreLuci/biographies/ faculty/djp3/

LocalCopy/compass03tr.doc; retrieved May 17, 2007

11. Hancke G, Kuhn M. An RFID distance bounding protocol. In: Proceedings of the First International Conference on Security and Privacy for Emerging Areas in Communications Networks: SecureComm 2005, Athens, Greece, 5-9 September 2005. Los Alamitos: IEEE Computer Society; 2005; pp 67-73

12. Arslan H, Ning Chen Z, Di Benedetto M. Ultra wideband wireless communication. New Jersey: WileyInterscience; 2006

13. Greiner C, Makimoto K, Suzuki M, Yamakawa M, Ashida N. Feasibility study of the integrated circuit tag monitoring system for dementia residents in Japan. American Journal of Alzheimer's Disease and Other Dementias 2007;22(2):129-136

14. Saito T, Shibata T, Wada K, Tanie K. Relationship between interaction with the mental commit robot and change of stress reaction of the elderly. In: Proceedings of the 2003 IEEE International Symposium on Computational Intelligence in Robotics and Automation, July 16-20, 2003; Kobe, Japan, volume 1. Piscataway: IEEE; pp 119-124

15. Kearns WD, Rosenberg D, West L, Applegarth S. An evaluation of stakeholder attitudes and expectations of technologies to manage wandering behavior in persons with dementia. Gerontechnology 2007;6(2):89-101

16. Korhonen I, Pärkkä J, Gils M van. Health monitoring in the home of the future. IEEE Engineering in Medicine and Biology Magazine 2003;22(3):66-74

17. Qian H, Loizou P, Dorman M. Phone-assistive devices based on Bluetooth technology for cochlear implant users. IEEE Transactions on Neural Systems Rehabilitation Engineering 2003;11(3):282-287

18. Albright F. Future shop. St. Petersburg Times 2007 (February 5): Section D:1

19. Coleman R. Improving the quality of life for older persons by design. In: Graafmans J, Taipele V, Charness N, editors. Gerontechnology: A sustainable investment in the future. Amsterdam: IOS 


\section{High-speed networking}

Press; 1998; pp 74-83

20. Struker J, Sackmann S, Muller G. Case study on retail customer communication applying ubiquitous computing. In: Bichler M, Chung J.-C, editors. Proceedings of the IEEE International Conference on e-commerce technology, CEC 2004, July 2004. Los Alamitos, CA: IEEE Computer Society; 2004; pp 42-48; http://ieeexplore.ieee.org/5/9218/29236/ 01319716.pdf?isnumber $=29236 \&$ prod=STD\&arnumber $=1319716 \&$ arnumber $=1319716 \&$ arSt $=+42 \&$ ared $=+48 \&$ arAuthor $=$ Struker $\% 2 C+\mathrm{J} . \%$ 3B+Sackmann $\% 2 \mathrm{C}+\mathrm{S} . \% 3 \mathrm{~B}+$ Muller\% 2C+G; retrieved May 17, 2007

21. Fogg B. Persuasive technology: Using computers to change what we think and do. New York: Morgan Kaufmann; 2002

22. Kearns WD, Fozard JL. Technologies to manage wandering. In: Nelson A, Algase $D$, editors. Safe and ethical approaches for wandering behaviors: A practice guide. New York: Springer; 2007 (in press)

23. Carraro GU, Cortes M, Edmark JT, Ensor JR. The Peloton bicycling simulator. In: Spencer SN, Roehl, B, editors. Proceedings VRML '98 : Third Symposium on the Virtual Reality Modeling Language, Monterey, California, February 16-19, 1998. New York: Association for Computing Machinery; 1998; pp 63-70

24. Bouma $H$, Harrington TL. Information and communication. In: Harrington TL and Harrington MK, editors. Gerontechnology: Why and how. Maastricht: Shaker; 2000; pp 139-164

25. Internet2. Ann Arbor: The Association; 2007. Internet2 middleware initiative; http://middleware.internet2.edu/; retrieved February 7, 2007

26. Hypponen M. Malware goes mobile. Scientific American, 2006;295(5):70-77

27. Internet2. Ann Arbor: The Association; 2007. Internet2 multicast working group; http://multicast.internet2.edu/; retrieved May 23, 2007

28. Fozard JL, Metter EJ, Brant LJ, Pearson JD, Baker GT. Goals for the next generation of longitudinal studies. In: Schroots JJF, editor. Aging, health and competence. Amsterdam: Elsevier; 1993; pp 35-52

29. Lawton M. Future society and technology. In: Graafmans JAM, Taipele V, Charness N. editors, Gerontechnology: A sustainable investment in the future. Amsterdam: IOS Press; 1998; pp 12-22

30. Kahana E. A congruence model of person-environment interaction. In: Lawton MP, Windley PG, Byerts TO, editors, Aging and the environment: Theoretical approaches. New York: Springer; 1982; pp 97-121

31. Baltes PB, Baltes MM. Psychological perspectives on successful aging: The model of selective optimization with compensation. In: Baltes PB, Baltes MM, editors, Successful aging: Perspectives from the behavioral sciences. New York: Cambridge University Press; 1990; pp $1-27$

32. Fozard JL, Popkin SJ. Optimizing adult development: Ends and means of an applied psychology of aging. American Psychologist 1978;33:975-989

33. Fozard JL. Impacts of technology on health and self-esteem. Gerontechnology 2005;4(2):63-76 